\title{
SPIRAL ARM PATCHES IN Sc AND SBc GALAXIES
}

\section{PRONIK and K. CHUVAEV}

Crimean Astrophysical Observatory, Nauchny, Crimea, U.S.S.R.

Multicolor observations of galaxies are being carried out at the prime focus of the 2.6-m Schajn telescope using an image intensifier and 6-9 color filters. The effective wavelengths of the bandpasses used are approximately $3600,3730,4400,4680,5090$, $5280,6090,6600$ and $7400 \AA$. The filters for 3730,5090 and $6600 \AA$ are centered on emission lines.

Observations of the Sc galaxies NGC 628, 4254, 5194 and SBc galaxies NGC 925, $1073,3359,4088$ and 7741 were made in 1965-1969.

The energy distributions for the central region and for the bright patches in the spiral arms of every galaxy have been determined. For absolute calibration extrafocal star images were used. The background light of the nearest spiral arm has been subtracted.

Most of the patches show H $\alpha$-emission (Pronik and Chuvaev, 1967, 1969, 1970, 1971a, b; Pronik, 1972).

Color indices in Tifft's system have been calculated and two-color diagrams (1-3), (3-4) for the central regions and the spiral arm patches are shown in Figures 1-4. From these figures one can see that:

(1) The color indices of spiral arm patches form a sequence.

(2) This sequence is a continuation of the blue end of the sequence for the central regions of Sc galaxies (Tifft, 1961, 1963, 1969).

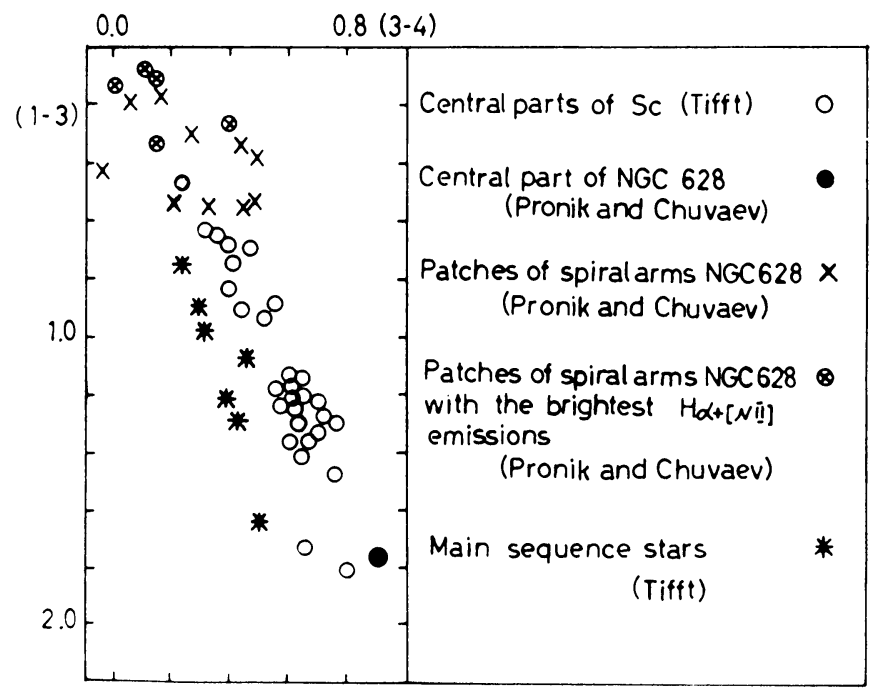

Fig. 1 . 
(3) The position of this sequence compared with that for the main sequence stars (Figure 1) shows that most patches contain not only blue stars but yellow ones as well.

(4) Very few of the bluest patches have colors like that of bluest stars.

(5) The reddest patches of all galaxies under consideration have colors like the bluest central regions of Sc galaxies.

(6) Galaxies having the bluest central regions have the bluest patches too.

(7) The reddening of the (1-3) colors of most of the patches in NGC 4254 (Figure 3) may be caused by interstellar dust absorption in the spiral arms of this galaxy.

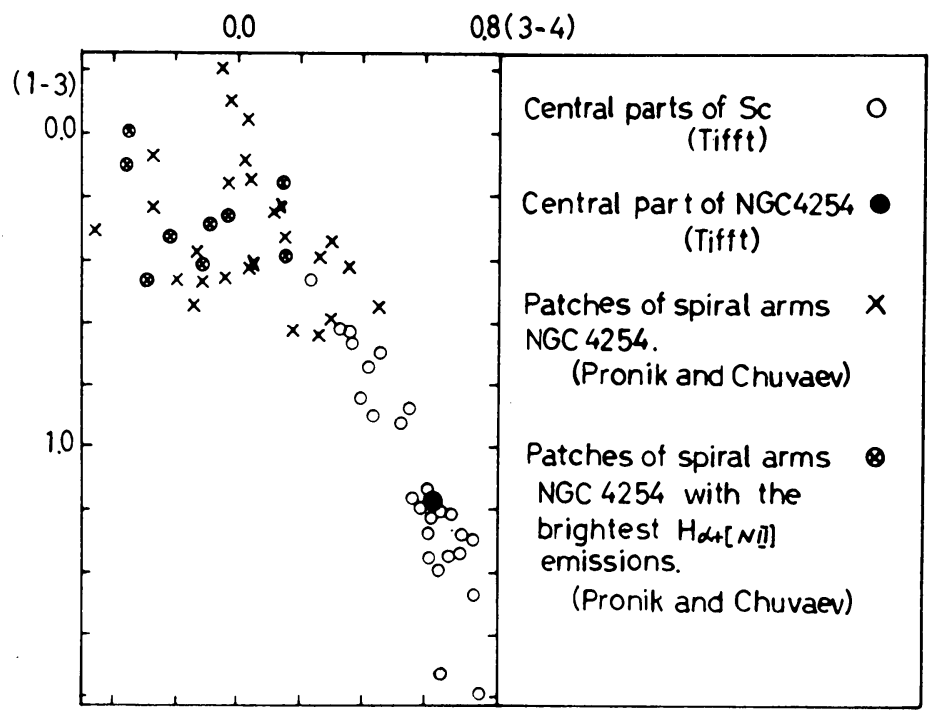

Fig. 2.

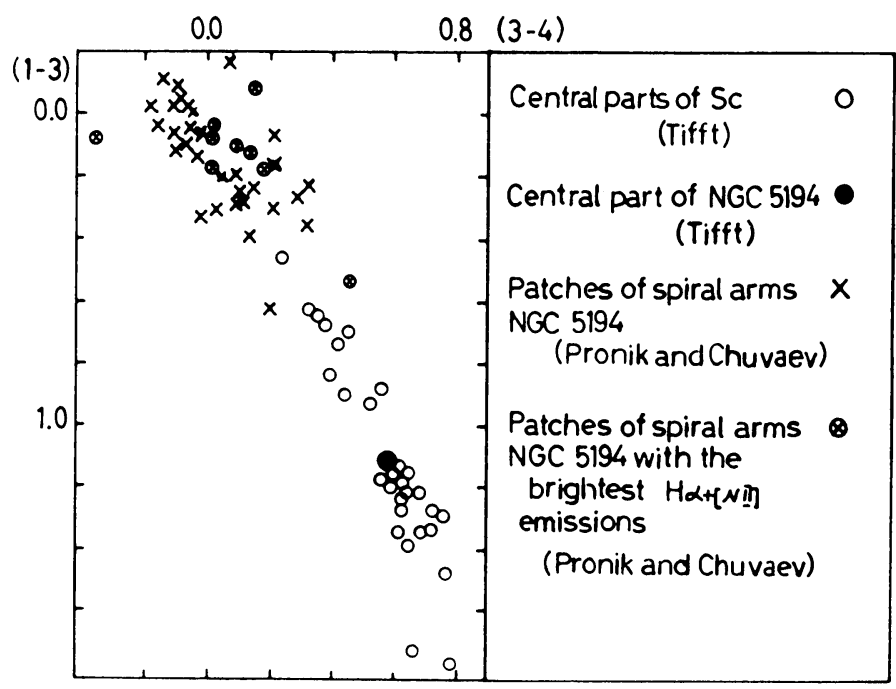

Fig. 3. 
(8) The central regions and spiral arm patches of five SBc galaxies studied (Figure 4) are bluer than those of the three Sc galaxies investigated.

Thus we can see that spiral arm patches or associations are not so young as the youngest stars, their ages are determined by the yellow stars present in the structures. Moreover one may suspect that the color-color sequences for spiral arm patches have an evolutionary nature. They are evolving into those for groups of stars having a spectral energy distribution like that of the central regions.

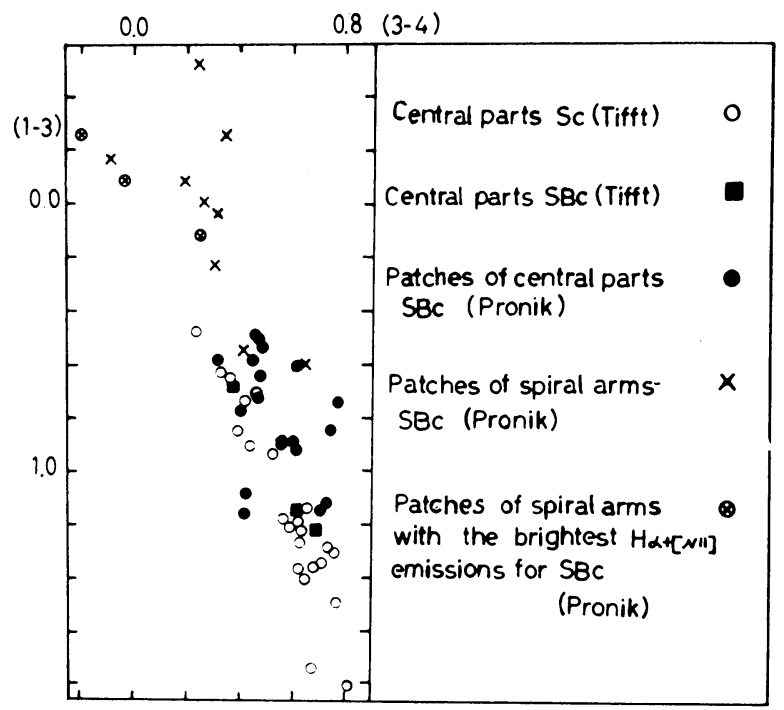

Fig. 4.

Figs. 1-4. Two-color diagrams for $\mathrm{Sc}$ and $\mathrm{SBc}$ galaxies.

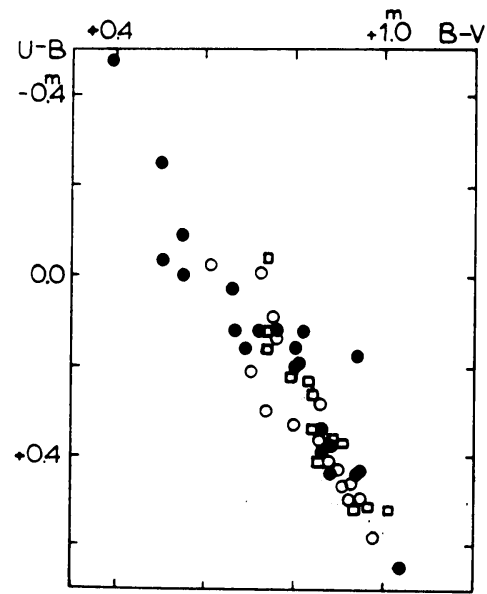

a

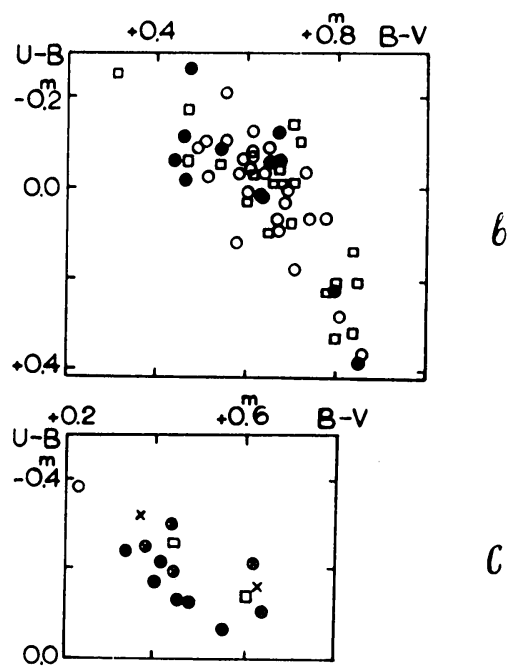

Fig. 5. Two colour diagram $(U-B),(B-V)$ for different morphological types (according to de Vaucouleurs): (a) 1-3 types, (b) 4-6 types, (c) 7-10 types. $\bigcirc=$ A galaxies, $\square=$ ABgalaxies, $O=$ B galaxies. 
In connection with point 8 consider Figure 5. This shows three two-color diagrams in the UBV system for $\mathrm{S}$ galaxies as a whole according to de Vaucouleurs and de Vaucouleurs (1964). From this figure one can see that SB galaxies are statistically bluer than the ordinary ones of the same morphological type. Among the latest types barred spirals predominate. It would be interesting to know with what kind of galaxy structures this effect is connected (bars, blue patches or both).

\section{Acknowledgement}

We are indebted to Mrs A. Bruns for detailed numerical work and for drawing the figures.

\section{References}

de Vaucouleurs, G. and de Vaucouleurs, A.: 1964, Reference Catalogue of Bright Galaxies, University of Texas Press, Austin.

Pronik, I. and Chuvaev, K.: 1967, Izv. Krymsk. Astrofiz. Obs. 38, 219.

Pronik, I. and Chuvaev, K.: 1969, Izv. Krymsk. Astrofiz. Obs. 40, 96.

Pronik, I. and Chuvaev, K.: 1970, in W. Becker and G. Contopoulos (eds.) 'The Spiral Structure of our Galaxy', IAU Symp. 38, 83.

Pronik, I. and Chuvaev, K.: 1971a, Izv. Krymsk. Astrofiz. Obs. 43, in press.

Pronik, I. and Chuvaev, K.: 1971b, Izv. Krymsk. Astrofiz. Obs. 44, in press.

Pronik, I.: 1972, Izv. Krymsk. Astrofiz. Obs. 45, in press.

Tifft, W.: 1961, Astron. J. 66, 390.

"Tifft, W.: 1963, Astron. J. 68, 301.

Tifft, W.: 1969, Astron. J. 74, 354. 